\title{
Analysis of disordered nonlinear domain statistics via second harmonic diffraction
}

C. Cojocaru, B. Wang, K. Switkowski, W. Krolikowski, M. Scalora, et al.

C. Cojocaru, B. Wang, K. Switkowski, W. Krolikowski, M. Scalora, J. Trull, "Analysis of disordered nonlinear domain statistics via second harmonic diffraction," Proc. SPIE 10683, Fiber Lasers and Glass Photonics: Materials through Applications, 106830E (17 May 2018); doi: 10.1117/12.2322627

SPIE. Event: SPIE Photonics Europe, 2018, Strasbourg, France 


\title{
Analysis of disordered nonlinear domain statistics via second harmonic diffraction
}

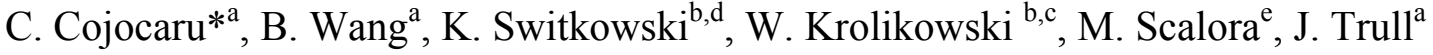 \\ ${ }^{a}$.Universitat Politècnica de Catalunya, Physics Department, Rambla Sant Nebridi 22, 08222

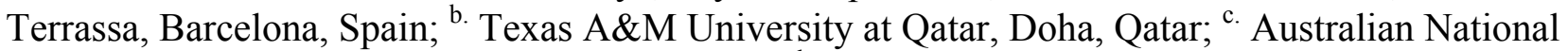

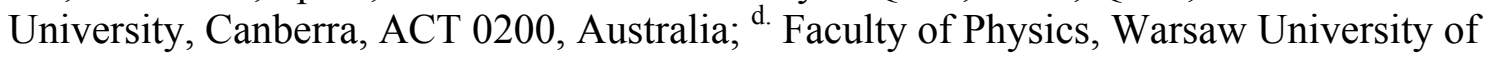 \\ Technology, Warsaw, Poland; ${ }^{\text {e. }}$ Charles M. Bowden Research Facility, AMRDEC-RDMR-WDS, \\ Redstone Arsenal, USA
}

\begin{abstract}
We present an indirect and non-destructive optical method for domain statistic characterization in disordered nonlinear crystals, having a spatially random distribution of ferroelectric domains with homogeneous refractive index. This method, based on a combination of numerical simulations and experimental measurements, analyses the wavelengthdependent second harmonic spatial distribution. We apply this technique to the characterization of two different random media, with drastically different statistical distributions of ferroelectric domains.
\end{abstract}

Keywords: nonlinear optics, harmonic generation, random nonlinear materials, nonlinear domain statistics, nondestructive optical method.

\section{INTRODUCTION}

It is well known that one dimensional (1D) structures with periodic inversion of the quadratic nonlinearity $\left(\chi^{2}\right)$ sign can compensate the phase mismatch $(\Delta k)$ of a particular nonlinear process. To this end, the spatial periodicity $(\Lambda)$ has to fulfill the condition $\Lambda=2 \pi / \Delta k$. This process can be understood by assuming that the spatial periodicity introduces a reciprocal lattice vector (RLV) $G=2 \pi / \Lambda$ that compensates the phase mismatch, so the condition $\Delta k+G=0$ holds. [1,2] This idea can be extended to more sophisticated nonlinear structures having 2D periodic modulation of the nonlinearity [3], where the number of reciprocal lattice vectors available increases and can simultaneously satisfy phase matching conditions for non-collinear propagation or multiple wavelengths. Later, quasi-periodic distributions provided an even broader RLV space $[4,5]$.

In this spirit, structures displaying homogeneous linear properties, but having a 2D random distribution of domains with inverted sign of $\chi^{2}$, further increase the RLV space by providing a continuous set of reciprocal vectors that span around a central value determined by domain size. Some as-grown ferroelectric crystals, as for example $\mathrm{SBN}, \mathrm{BaTiO}_{3}$ and $\mathrm{LiNbO}_{3}$, possess this kind of domain distribution and provide phase matching over a broadband range of wavelength. [6, 7]. These crystals show a needle-like structure of domains oriented along the optical axis with a set of RLV confined in the plane perpendicular to this axis. When a fundamental beam propagates in this plane, the crystal emits a SH signal over the whole plane [8]. Planar SH generation of these crystals has been successfully used for different applications such as characterization of ultrashort optical pulses [9-11], cascaded third harmonic generation [12] and nonlinear domain visualization [13].

The angular spreading of the planar SH emission is strongly dependent on the RLV distribution, which in turn depends on the particular domain size and distribution within the crystal. Any change in the domain pattern will be reflected in the transverse distribution of the SH intensity. Its characterization could bring information about the domain pattern parameters, difficult to be obtained by imaging methods since there is no linear refractive index contrast between them and the domain size is very small (between $100 \mathrm{~nm}$ and few $\mu \mathrm{m}$ ).

*crina.maria.cojocaru@upc.edu; phone +34 937398571; https://donll.upc.edu/en 
Based on the model suggested by Le Grand et al. [14], SH diffraction has been used to forecast the domain statistics of random nonlinear crystals $[15,16]$. However, even for the natural (as grown) distributed domain statistics the assumed model yields some discrepancies between the theoretical fits and the experimental results [17]. Therefore, a more precise 2D method for detecting domain statistics is well worth exploring.

In this work we present a combined experimental and numerical study of the angular distribution of the planar $\mathrm{SH}$ emission in different types of random media. We demonstrate that the emitted SH pattern variation as a function of fundamental wavelength provides relevant information about the statistics of the ferroelectric domain pattern in the crystal. We use a numerical model based on a 2D, split-step fast Fourier transform beam propagation method to verify and complement the experimental observations.

\section{MODELS AND EXPERIMENTAL RESULTS}

Second harmonic scattering from materials with a random distribution of $\chi^{2}$ has been study by different authors. Among them, Dolino et al. [18] showed that the intensity of the SH emission should be proportional to the square modulus of the Fourier transform of the nonlinear polarization distribution in the material, which corresponds to the RLV distribution provided by the structure. This indicates that $\mathrm{SH}$ will be emitted with a higher intensity in a particular direction within the crystal if RLV compensates for the phase mismatch in that direction. Figure 1(b) shows schematically how the phase mismatch compensation takes place for a particular SH emission angle. A perfectly homogeneous distribution of $G$ vectors, spanning all possible directions in the plane, should give rise to a homogeneous planar SH signal as shown schematically in Figure 1(a). Any deviation from this ideal frame should be reflected in the angular dependence of the observed SH pattern.

(a)

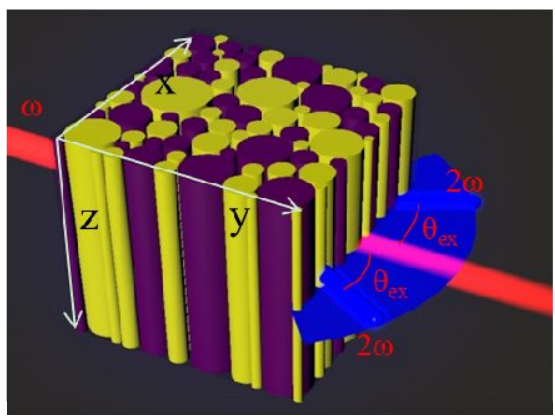

(b)

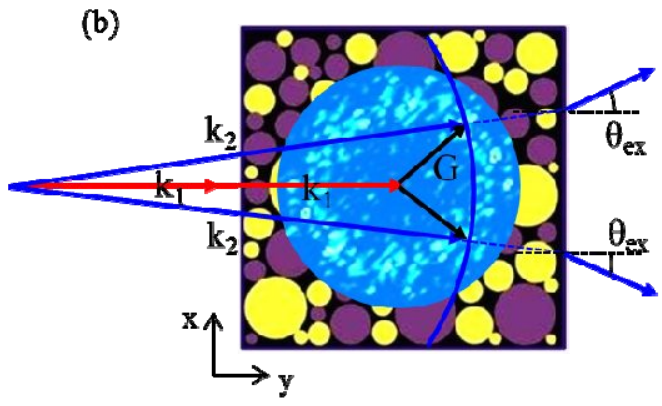

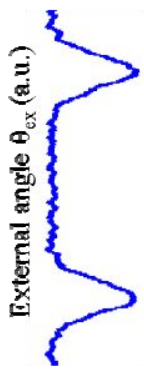

Figure 1. (a) Schematic representation of the second harmonic generation in the SBN crystal (b) The 2D QPM geometry in $\mathrm{SH}$ diffraction process and the external angle emission pattern.

In a later work Le Grand et al. [14] obtained an analytical expression for the observed SH intensity pattern. They calculated the Fourier transform corresponding to a normal distribution of domain sizes around a particular central value of domain diameter, $\rho_{o}$, consistent with a RLV vector $\left|\vec{G}_{m}\right|=\pi / \rho_{o}$ and a given mean standard deviation $\sigma$;

$$
I(2 \omega) \propto \frac{I(\omega) d_{e f f} k_{\omega}^{2}}{n_{\omega}^{4} \cdot n_{2 \omega}} \cdot \frac{4 L}{G^{2}} \cdot \frac{1-e^{-G^{2} \sigma^{2}}}{1+e^{-G^{2} \sigma^{2}}+2 \cos \left(G \rho_{o}\right) e^{-G^{2} \sigma^{2} / 2}} .
$$

Here, $I(\omega)$ is the intensity of the fundamental wave, $d_{e f f}$ the effective quadratic nonlinear coefficient which depends on material parameters and interaction geometry, and $G=|\Delta k|$ is the absolute value of the phase mismatch. The last term represents the effect of disorder in the domain distribution. A typical emission pattern corresponding to equation (1) is shown in Figure 2(a). A structure with well-defined average domain diameter $\rho_{o}$ and small standard deviation gives rise to an emission pattern having two well-resolved maxima, which correspond to the emission angles where the phase mismatch is compensated by the particular RLV provided by $\left|\vec{G}_{m}\right|$. For the kind of normal dispersion found in typical 
ferroelectric crystals, one can show that a change in wavelength results in a change of the angular emission because the $G$ vector distribution remains unaltered (fixed by the crystal). However, the phase matching condition change due to material dispersion. Figure 2 (b) shows an increased emission angle when the wavelength increases.

(a)

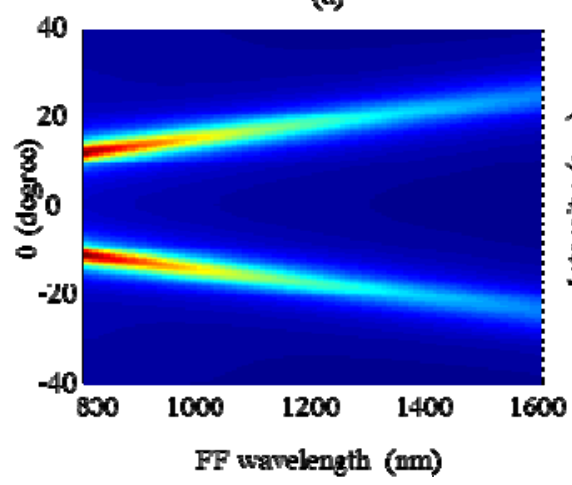

(b)

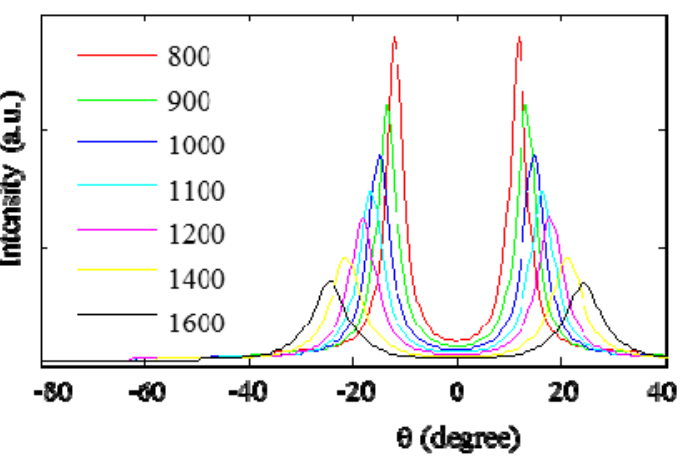

Figure 2 (a) SH emission over a plane perpendicular to the optical axis for a structure made with normal distribution of domains with a mean value of $0.9 \mu \mathrm{m}$ and standard deviation $\sigma=0.3 \mu \mathrm{m}$ as a function of the incident fundamental wavelength. The $0^{\circ}$ direction corresponds to emission in the forward direction of the incident beam. (b) Calculated angle of maximum $\mathrm{SH}$ emission as a function of wavelength.

In this work we study two different nonlinear crystals with disordered domain structure: sample 1 is a Calcium Barium Niobate (CBN) crystal which is a typical as-grown crystal with neither temperature nor electric field treatment; sample 2 is a Strontium Barium Niobate (SBN) crystal which was partially poled by exposing it to a variable electric field to change its domain distribution in a non-prescribed way. For both samples we measured the SH pattern emitted for two different fundamental wavelengths: $800 \mathrm{~nm}, 1064 \mathrm{~nm}$ and 1200nm. The collimated fundamental laser beam impinged on the crystal at normal incidence. The crystal optical axis $(Z)$ was placed vertical such that the SH pattern was emitted in a horizontal plane. A rotating arm, centered on the crystal position allowed us to measure the SH as a function of the angle with respect to the direction of propagation of the fundamental beam. A photodiode with a 200-micron pinhole diaphragm was used to measure the SH signal in each particular direction. Ordinary or extraordinary polarization of the fundamental beam was selected using a half-wave plate placed in front of the crystal. The SH polarization is always extraordinary since the nonlinear susceptibility matrix of these crystals allows for both ee-e and oo-e interaction [19].

The experimental results are shown in Figure 3, where we plot the measured angular distribution of the SH emitted at $400 \mathrm{~nm}$ and at $532 \mathrm{~nm}$ for the as-grown CBN (sample 1) and the artificially poled SBN crystal (sample 2). These results clearly show a drastic difference in the angular SH intensity patterns for both crystals. In the CBN crystal the SH is emitted in a broad angular range up to $60^{\circ}$; the emission patterns for both fundamental wavelengths are similar. In contrast, the SBN sample leads to a much narrower SH angular emission with two clear maxima whose separation is sensitive to the fundamental wavelength. Moreover, an increasing wavelength causes these maxima to move in a direction opposite to that predicted by the Le Grand model illustrated in Figures 2: longer wavelengths correspond to smaller angle. 
(a) Sample 1

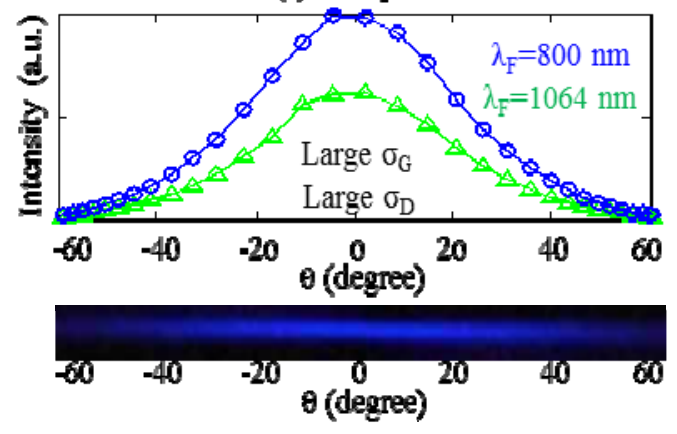

(b) Sample 2

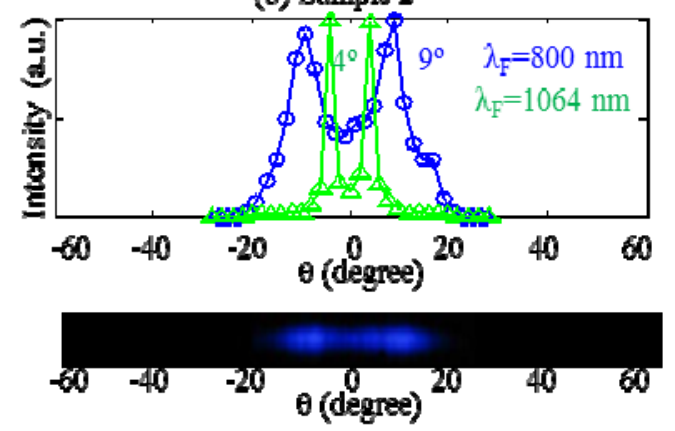

Figure 3. SH emission pattern measured from sample $1(\mathrm{CBN})$ and sample $2(\mathrm{SBN})$ for two different fundamental wavelengths: $800 \mathrm{~nm}$ (blue line; circles) and $1064 \mathrm{~nm}$ (green line; triangles). Below each figure we show a picture of the SH pattern at $400 \mathrm{~nm}$ for each sample, recorded with a CCD camera placed in front of the crystal.

The SH angular distribution from the CBN (sample 1) represents the emission pattern from a medium with statistical domain and RVL distribution with large standard deviation $\sigma$. As $\sigma$ increases, the initially two well resolved SH maxima shown in Figure 2 start to broaden and subsequently merge forming a single-peaked, broad intensity distribution similar to that of sample 1. A more detailed study of this effect can be found in ref. [15]. However, the behaviour of the SBN crystal (sample 2) cannot be reproduced using the Le Grand model, thus highlighting its limits.

\section{RESULTS AND DISCUSSIONS}

To get an insight of these experimental results we have developed and implemented a numerical model that simulates the beam propagation and SH generation in 2D random domains samples. A fundamental beam propagates in the y direction and SH diffraction emission occurs in the x-y plane (see Figure 2). Since the ferroelectric domains have needle-like shape along the $\mathrm{z}$ axis and the random structure lies in the $\mathrm{x}-\mathrm{y}$ plane, we adopt a 2D solution to simulate the SH generation based on the split-step fast Fourier transform (FFT) algorithm modified to handle the propagation of pulses of arbitrary duration [15]. Regarding the 2D structures, we assumed individual domains in the form of rods with a circular transverse profile; after selecting a mean domain size and a certain distribution and filling fraction, a given configuration is randomly generated and randomly placed in a rectangular area representing the size of the sample; the number of domains that takes part in the nonlinear process should be large compared to the actual size of the input optical beam.

In the 2D domain pattern simulation for sample 1, domain size was assumed to have a Gaussian distribution with the experimentally retrieved mean diameter $\left(D_{\max }=2.0 \mu \mathrm{m}\right)$. This assumption is based on the analysis of real domain image of the crystals obtained by nonlinear microscopy [20]. We have considered different values of the Gaussian distribution variance $(\sigma)$ until we have obtained the same angular distribution of the generated $\mathrm{SH}$ as in the experimental results. Figure 4 (a) contains a typical generated 2D domain structure in the $x-y$ plane, simulating sample 1 . The white and black regions represent domains with opposite sign of the nonlinearity. Figure 4 (b) is the corresponding Fourier spectrum of the domain structure in reciprocal space. The majority of reciprocal vectors are gathered around a broad ring with radius $G_{\max }=\pi / D_{\max }=1.5 \mu \mathrm{m}^{-1}$.

To simulate the artificial poled sample 2, we have consider that the domains with positive $\chi^{(2)}$ have very low filling factor (around 6\%) and they are placed on a homogeneous negative $\chi^{(2)}$ background. We have simulated different Gaussian distribution around the diameter mean value of $1 \mu \mathrm{m}$ until we have obtain the same SH angular distribution as in the experiment. The simulated 2D domain structure in the $x-y$ plane is shown in Figure 4 (c) where a Gaussian distribution of domains with a mean size of $D_{\max }=0.9 \mu \mathrm{m}$ has been assumed. Figure 4 (d) is the corresponding Fourier spectrum of the domain structure in reciprocal space. The majority of reciprocal vectors are gathered around a narrow ring with radius $\boldsymbol{G}_{\boldsymbol{m a x}}=\boldsymbol{\pi} / \boldsymbol{D}_{\boldsymbol{m a x}}=3.5 \mu \mathrm{m}^{-1}$. 
(a)

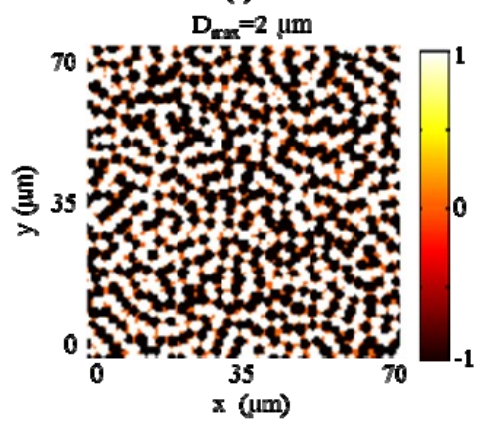

(c)

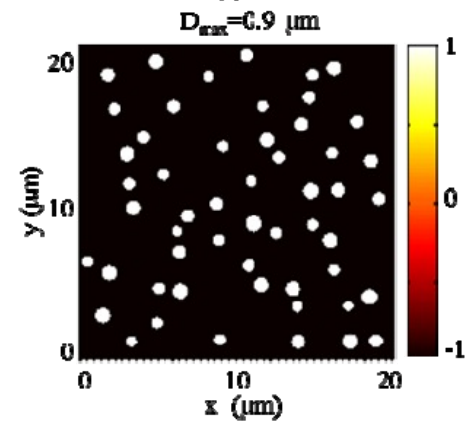

(b)

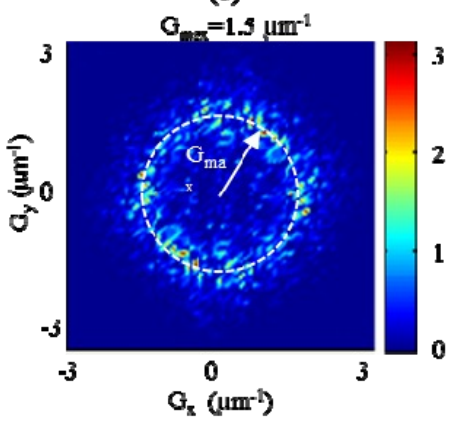

(c)

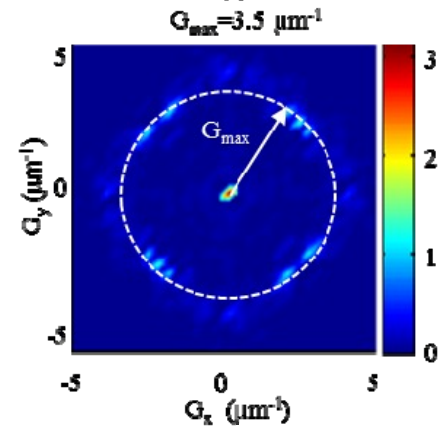

Figure 4. (a) 2D domain pattern simulation in real space for the as grown CBN crystal (sample 1); (b) the corresponding Fourier spectrum in the reciprocal space for sample 1; (c) 2D domain pattern simulation in real space for the artificial poled SBN crystal (sample 2) and (d) the corresponding Fourier spectrum in the reciprocal space for sample 2.

A typical result of the propagation code is shown in Figure 5 for a particular realization close to Le Grand type distribution similar to that shown in Figure 4(a). Figure 5(a) represents the far field distribution in air (equivalent to the spatial transverse or angular spectrum) as a function of the propagation distance inside the crystal. This time the fundamental wavelength was set to $1200 \mathrm{~nm}$. The far-field angular distribution profile at the maximum propagation distance is plotted in Figure 5(b). The SH emission in the form of well resolved maxima is clearly observed as expected.

(a)

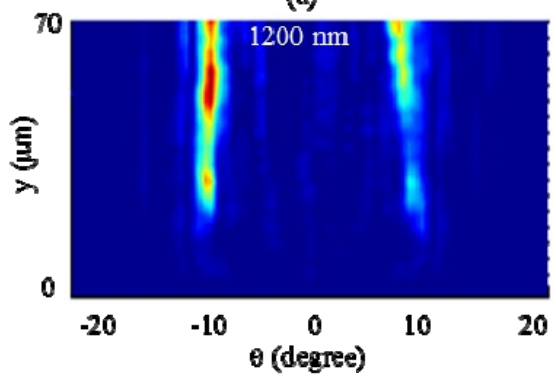

(b)

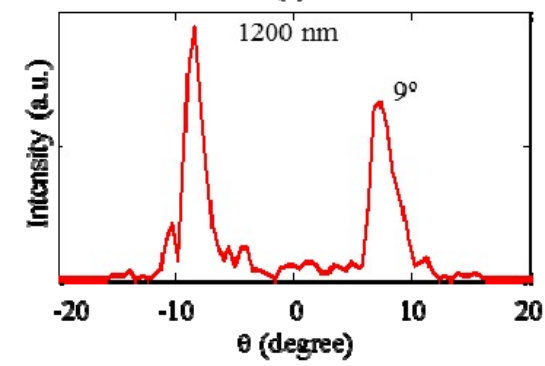

Figure 5. (a) Far field SH angular pattern as a function of propagating distance. (b) Far field intensity distribution after $x=70$ $\mu \mathrm{m}$ propagation distance within the crystal.

Figure 6 shows a comparison between our experimental results at $800 \mathrm{~nm}$ and $1200 \mathrm{~nm}$ for both oo-e interaction and ee-e interaction (upper part) and the simulated far-field angular distribution (lower part). Simulation and experiment are in good agreement, showing that the process of averaging over different realizations can be an efficient way to simulate the large variance distribution. 

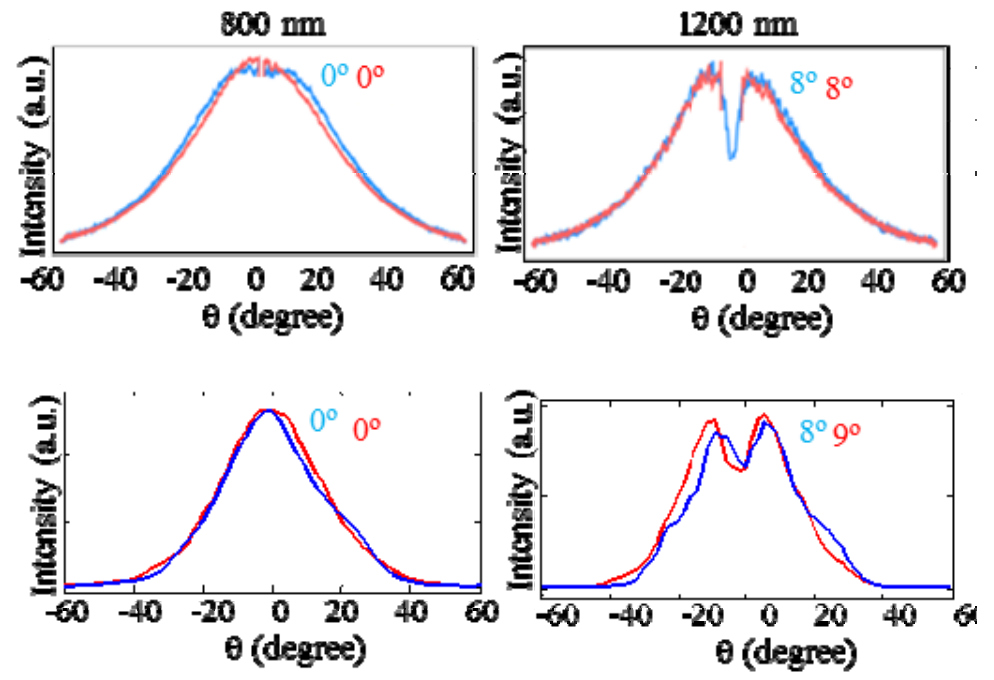

Figure 6. Comparison between the experimental results (top) and numerical simulations (bottom) for as-grown CBN crystal at two different wavelengths for the domain distribution shown in Fig. 4 (a).

The results for sample 2 show the combined effects of domain distribution and nonlinear background on the SH intensity angular distribution. From the far-field angular distribution results we surmised that the nonlinear domain distribution dominates the parametric process between $800-1200 \mathrm{~nm}$. Figure 7 shows a comparison between our simulated far-field angular distribution and the experimental results for both oo-e interaction (blue line) and ee-e interaction (red line). The simulation results are in good agreement with the experimental results.
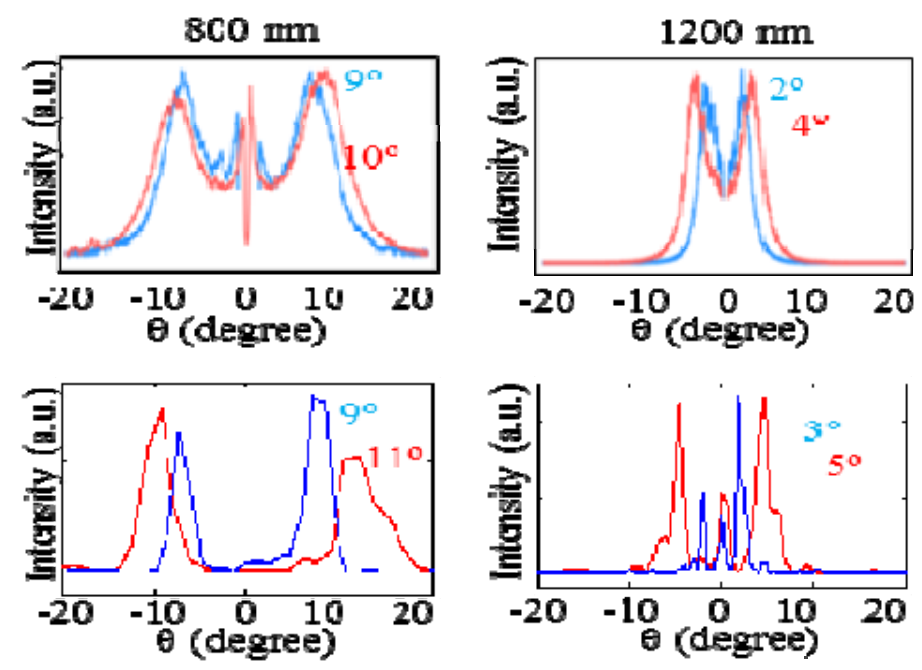

Figure 7. Comparison between the experimental results for SBN crystal, sample 2 (top) and the numerical simulations (bottom) at different wavelengths for the domain distribution shown in Fig. 4(c)

\section{CONCLUSIONS}

We demonstrate an indirect non-destructive optical method for the characterization of nonlinear domain statistics based on the analysis of the SHG intensity angular distribution. The method is based on a combination of experimental measurements and numerical simulations. We begin by experimentally measuring a SH diffraction pattern from real 
crystals. Then we designed a 2D domain structures and numerically simulate the propagation through them using a spectral method, and find good agreement between our simulations and the experimental data. From these we deduce that our domain designs are realistic. The method was implemented experimentally and tested in crystals with different types of domain distributions. The analysis of the dependence of the angular distribution of SHG on the fundamental beam wavelength informs us about complicated domain structures. This method could be used for real time monitoring of the unknown domain distribution during poling or crystal growing process.

Authors acknowledge financial support by: Spanish Ministerio de Educación y Ciencia (project FIS2011-29734-C0201), US Army ACQ-RDECOM (grants W911NF-12-1-0201 and W911NF-16-1-0563) and Australian Research Council and Qatar National Research Fund (NPRP8-246-1-060).

\section{REFERENCES}

[1] J.A. Armstrong, N. Bloembergen, J. Ducuing and P.S. Pershan, "Interactions between light waves in nonlinear dielectrics" Phys. Rev. A 127, 1918-1939 (1962).

[2] M.M. Fejer, G.A. Magel, D.H. Jundt and R.L. Byer, "Quasi-Phase-matched Second harmonic Generation: Tuning and Tolerances" IEEE Journal Quant. Electron, QE-28 (11), 2631-2654 (1992).

[3] V. Berger "Nonlinear Photonic Crystals", Phys. Rev. Lett. 81(19), 4136-4139 (1998).

[4] N.G.R. Broderick, G.W. Ross, H.L. Offerhaus, D.J. Richardson and D.C. Hanna, "Hexagonally Poled Lithium Niobate: A two-Dimensional Nonlinear Photonic Crystal," Phys. Rev. Lett, 84, 4346-4349 (2000).

[5] Y. Sheng, J. Dou, B. Ma, B. Cheng and D. Zhang, "Broadband efficient second harmonic generation in media with short-range order," Appl. Phys. Lett. 91, 011104 (2007).

[6] R.C. Miller "Optical harmonic generation in single crystal BaTiO3," Phys. Rev. A, 134 (5), A313-A1319 (1998).

[7] R.V. Gainutdinov, T.R. Volk, O.A. Lysova, I.I. Razgonov, A.L. Tolstikhina and L.I. Ivleva, "Recording of domains and regular domain patterns in strontium-barium-niobate crystals in the field of atomic force microscope" Applied Physics B 95, 505-512 (2009).

[8] A.R. Tunyagi, M. Ulex and K. Betzler, "Noncollinear Optical frequency Doubling in Strontium Barium Niobate," Phys. Rev. Lett. 90, 243901-1/243901-4 (2003).

[9] J. Trull, I. Sola, B. Wang, A. Parra, W. Krolikowski, Y. Sheng, R. Vilaseca and C. Cojocaru "Ultrashort pulse chirp measurement via transverse second-harmonic generation in strontium barium niobate crystal," Appl. Phys. Lett. 106, 221108 (2015).

[10] A.S. Aleksandrovsky, A.M. Vyunishev, A. I. Zaitsev, A.A. Ikonnikov and G.I. Pospelov, "Ultrashort pulses characterization by nonlinear diffraction from virtual beam" Appl. Phys. Lett. 98, 061104 (2011).

[11]B. Wang, C. Cojocaru, W. Krolikowski, Y. Shens and J. Trull, "Transverse single shot cross-correlation scheme for laser pulse temporal measurement via planar second harmonic generation" Optics Express 24, 22210, 2016.

[12] W. Wang, V. Roppo, K. Kalinowski, Y. Kong, D.N. Neshev, C. Cojocaru, J. Trull, R. Vilaseca, K. Staliunas, W. Krolikowski, S.M. Saltiel and Yu. Kivshar, "Third-harmonic generation via broadband cascading in disordered quadratic nonlinear media," Opt. Express 17 (22) 20117 (2009).

[13] Y. Sheng, A. Best, H. Butt, W. Krolikowski, A. Arie and K. Koynov, "Three-dimensional ferroelectric domain visualization by Cerenkov-type second harmonic generation," Opt. Express 16, 16539 (2010)

[14] Y. Le Grand, D. Rouede, C. Odiu, R. Aubry and S. Mattauch "Second-harmonic scattering by domains in $\mathrm{RbH}_{2} \mathrm{PO}_{4}$ ferroelectrics", Opt. Commun. 200, pp. 249-260 (2001).

[15] V. Roppo, W. Wang, K. Kalinowski, Y. Kong, C. Cojocaru, J. Trull, R. Vilaseca, M. Scalora, W. Krolikowski, and Yu. Kivshar, "The role of ferroelectric domain Structure in second harmonic generation in random quadratic media", Opt. Express18, 4012-4022 (2010).

[16] M. Ayoub, J. Imbrock and C. Denz "Ferroelectric domain diagnostics near the phase transition by Cerenkov second-harmonic generation" Opt. Express 19, 11340-11354 (2011).

[17] M. Ayoub, J. Imbrock and C. Denz, "Second harmonic generation in multi-domain $\chi^{2}$ media: from disorder to order," Opt. Mat. Express 7, 3448-3455 (2017). 
[18] G. Dolino, J. Lajzeriwicz and M. Vallade, "Second-harmonic Light Scattering by Domains in Ferroelectric Triglycine Sulfate," Phys. Rev. B. 2, 2194-2200 (1976).

[19] J. Trull, C. Cojocaru, R. Fischer, S.M. Saltiel, K. Staliunas, R. Herrero, R. Vilaseca, D.N. Neshev, W. Krolikowski and Y.S. Kivshar, "Second-harmonic parametric scattering in ferroelectric crystals with disordered nonlinear domain structures," Opt. Express 15, 15868-15877 (2007).

[20] B. Wang, K. Switowski, C. Cojocaru, V. Roppo, Y. Sheng, M. Scalora, J. Kisielewski, D. Pawlak, R. Vilaseca, H. Akhouayri, W. Krolikowski and J. Trull, "Comparative analysis of ferroelectric domain statistics via nonlinear diffraction in random nonlinear materials" Opt. Express 26 (2) 1083-1093 (2018). 